\title{
Electrofisiología $\mathrm{y}$ eficiencia intelectual
}

\section{Jean-Claude Grubar}

Universidad de Lille III

Traducción de Pedro Aller

\section{INTRODUCCION}

Los progresos conjuntos de la fisiología, la bioquímica, la psico-fisiología y la etología ofrecen la expectativa de llegar a un nuevo enfoque y a una nueva concepción de la inteligencia.

Este nuevo enfoque cuestiona el dualismo cuerpo-espíritu, el dualismo organismo-actividades cognitivas superiores. Las actividades cognitivas superiores tienen un substrato fisiológico; el espíritu tiene su origen en el cuerpo, la inteligencia deriva del instinto.

Investigar las bases fisiológicas de la inteligencia consiste, actualmente, en recoger cierto número de índices fisiológicos correlativos a las actividades cognitivas, tomar índices de estados que favorecen tales actividades.

La electroencefalografía (EEG) ofrece un índice digno de tenerse en cuenta, el sueño es un estado cuya exploración abre interesantes perspectivas.

Lo mismo que ocurre en otros muchos dominios de la psicofisiología, el enfoque patológico es el más utilizado: el conocimiento de la inteligencia implica el conocimiento de sus deficiencias, es decir, de la debilidad mental.
La hipótesis general subyacente a los trabajos realizados en este campo es la de que hay una relación entre el sueño y las actividades cognitivas; es la hipótesis «sleep-cognition» propuesta por Hughlings Jackson (1932). Cuando se sitúa en el marco del enfoque patológico, esta hipótesis establece que los déficits de sueño reflejan los deterioros de las actividades cognitivas (o son la causa de ellos).

\section{EEG e INTELIGENCIA}

El descubrimiento de la electroencefalografía, por Hans Berger, en 1929, le: vantó muchas esperanzas, uno de cuyos extremos era el de una medida objetiva de la inteligencia.

En esta perspectiva y con este fin, muchos trabajos han tratado de establecer un paralelo entre los aspectos morfológicos del EEG y el nivel intelectual. Los primeros fueron los de Lyndsley (1938) y Henry (1944). Los aspectos morfológicos más estudiados han sido: la frecuencia del ritmo alfa, la tasa de alfa en las zonas occipital, parietal y rolándica, el índice de alfa y la amplitud (voltaje) de la señal. Ni Lindsley ni Henry encon- 
traron correlaciones entre el CI y la frecuencia o estructura del ritmo alfa.

Posteriormente, los trabajos más completos fueron los de Netchine (1959), Netchine y Lairy (1960) y Bogel y Broverman (1964).

Después de realizar un estudio con 209 niños de 5 a 12 años, distribuidos en tres niveles intelectuales, Netchine y Lairy llegaron a la conclusión de que la frecuencia y dispersión del alfa occipital aumentan, por término medio, al aumentar el nivel intelectual. Además encontraron que los niños del grupo con $\mathrm{CI}$ igual o inferior a 75 , presentaban ritmos theta en mayor grado que los niños normales de la misma edad. La comparación de los ritmos occipitales y parietales les llevó a concluir que la diferencia entre ellos es más importante en los niños de nivel intelectual alto.

Vogel y Broverman también llegaron a la conclusión de que hay una relación entre la inteligencia y los aspectos morfológicos del EEG, especialmente en lo que se refiere al ritmo alfa occipital.

La presencia de ondas lentas en el EEG de vigilia está asociada con un desarrollo mental insuficiente $\mathrm{y}$ con la debilidad mental (Müller-Küpper y Vogel, 1965). Estos resultados se vieron confirmados por los de Blum y cols. (1973) que consideran que la presencia de ondas lentas constituye un pronóstico de evolución desfavorable.

Netchine (1967) estima que cuanto más elevado es el cr, más complejo resulta el EEG.

Los trabajos de este tipo no siempre han permitido obtener conclusiones tan claras: hay otros trabajos que no permiten obtener resultados significativos; Hill (1963), por su parte, presenta resultados opuestos.

En una revisión crítica de 1966, Ellingston estimaba que es imposible esta- blecer con certeza significativa ningún tipo de relación entre el EEG y las funciones complejas como la inteligencia, punto de vista que ya había adelantado Gastaut en 1960.

Pero este enfoque, que puede calificarse de estático, puede sustituirse por un enfoque dinámico, que tenga en cuenta la reactividad particular de los sujetos. En esta perspectiva, que es la única justificable (después de la obra de Duffy, 1962), el EEG ya no se considera índice de un estado permanente, sino testigo objetivo de la reactividad del sistema nervioso central a las influencias del medio.

El enfoque se basa en la hipótesis de que los individuos tienen una reactividad diferencial al medio, en función de su personalidad y de su nivel intelectual.

Las técnicas dél bloqueo alfa (alpha blocking) y de los potenciales evocados son las más utilizadas para dar cuenta de esa reactividad.

El substrato del bloqueo alfa es la desincronización del EEG, es decir, la clásica reacción de detención. Lo que se mide es la duración del intervalo entre el último huso alfa precedente al estímulo y el primero consecutivo a la reacción de detención.

Algunos trabajos, como los de Baker y Lodge (1967), Polikanina y Sergeeva (1967), Smith (1967), Voronin y cols. (1970), que investigaban la inhibición de la respuesta de orientación (objetivada por el bloqueo de alfa) consecutiva a la repetición de estímulos sonoros, han demostrado que el ritmo alfa se reinstala con más rapidez en sujetos normales que en mongólicos de la misma edad cronológica.

El potencial evocado es una secuencia de ondas consecutivas a una estimulación y se considera como la respuesta eléctrica de las zonas receptoras a dicha estimulación. Cualquier alteración de la morfo- 
logía de los potenciales evocados constituye un indicador de ciertas alteraciones funcionales de los mecanismos de transmisión e integración cerebral (Vanzulli y cols., 1960).

La latencia de los potenciales evocados indica la velocidad de transmisión y de integración. Chalke y Ertl (1965) y posteriormente Ertl $(1966,1969)$ y Ertl y Shafer (1969) han demostrado que se da una relación inversa entre la latencia de los potenciales evocados y el nivel intelectual: los cr débiles se asocian a latencias más elevadas. Ertl y sus colaboradores estiman que una latencia corta es índice de eficacia neurológica y, por tanto, un reflejo de la inteligencia.

También se ha correlacionado con la inteligencia la riqueza en armónicos de los potenciales evocados, índice de la repercusión cortical de làs estimulaciones: los potenciales evocados visuales de los individuos brillantes (desde el punto de vista intelectual) son más ricos en armónicos que los de los individuos menos brillantes (Rhodes y cols., 1969 a y b).

Sin embargo, en la revisión de Callaway (1973) sobre las relaciones entre los potenciales evocados y la inteligencia, la conclusión obtenida es más bien escéptica.

Estos trabajos, como los que se referían a las relaciones entre la morfología del EEG y la inteligencia, llegan a resultados contradictorios de los que resulta difícil extraer conclusiones definitivas.

Los resultados contradictorios obtenidos pueden deberse al hecho de que se han obtenido a partir del estudio de secuencias electroencefalográficas cortas; sería más pertinente sustituirlos por registros largos y continuados. Es indispensable tomar en consideración la influencia del factor «tiempo» en los registros electroencefalográficos, ya que este factor da cuenta de las variaciones de la actividad del sistema nervioso central.
Netchine y Lairy (1960) nos los recuerdan: el EEG refleja una actividad que varía considerablemente en el curso del nictámero, activación cuya relación con las actividades cognitivas nadie niega.

Es posible afirmar que las alteraciones de la organización nictameral de la activación están relacionadas con problemas comportamentales y déficits de las actividades cognitivas.

Todas las actividades psicológicas se basan en dos clases de funciones complementarias: las de registro y las de tratamiento de la información. La vertiente cognitiva de las actividades psicológicas consiste en el tratamiento de las. informaciones registradas en forma de estímulos tan numerosos momo variados, y en su integración a las estructuras previamente elaboradas. Podemos admitir, siguiendo a Jackson y a la mayor parte de los psicofisiólogos, que el registro y el tratamiento de la información tienen lugar en momentos diferentes y privilegiados del nictámero, organizados, sin duda, con arreglo a un ritmo circadiano.

En el curso del nictámero, el cerebro pasa por dos estados fundamentales: el de vigilia, en el que está sometido a una estimulación exógena (momento privilegiado para el registro de la información) y el de sueño, en el curso del cual se efectuaría el tratamiento de la información. Este tratamiento estaría ocasionado por una verdadera activación endógena del sistema nervioso central durante el sueño. Esta formulación constituye el substrato de la hipótesis «sueño-actividades cognitivas».

El estado de vigilia de los débiles mentales sólo se ha estudiado a partir del registro de secuencias electroencefalográficas breves. El empleo de registros más largos permitiría, como ya hemos señalado antes, aclarar los resultados contradictorios con los que contamos actualmente. De cualquier modo, y tenien- 
do en cuenta lo que hemos dicho, es más prudente no sacar conclusiones demasiado precoces sobre un posible déficit fisiológico del registro y la recepción de la información en los débiles mentales.

Por el contrario, el estudio poligráfico del sueño, es decir, de la vertiente del tratamiento e integración de la información —en el que sí se tiene en cuenta el factor «tiempo»- puede proporcionarnos resultados y conclusiones menos controvertidos y más fiables.

\section{LOS DOS SUENOS}

Fue Hughlings Jackson quien propuso por primera vez la hipótesis de que hay una relación entre el sueño y las actividades cognitivas $y$, más específicamente, entre el sueño y la memoria. Según este autor, el sueño tendría la función de borrar los recuerdos superfluos y jugaría un papel en la solución de problemas.

El desarrollo y la mejora de las técnicas electrofisiológicas ha ocasionado una renovación considerable del interés por el sueño $\mathrm{y}$ ha permitido tratar al sueño no sólo como un estado opuesto al de vigilia, sino como un parámetro simple con valor de variable dependiente $o$ independiente. De este modo, el enfoque experimental ha venido a sustituir a las especulaciones de antaño, permitiendo la verificación de hipótesis interesantes, especialmente la de Jackson.

El descubrimiento de dos estados de sueño por Aserinski y Kleitman (1953) fue determinante. Este descubrimiento venía a completar felizmente el enfoque psicológico de los niveles de profundidad del sueño (Loomis y cols., 1937).

Los registros continuados del sueño nocturno, del adormecimiento al despertar, con derivaciones electroencefalográficas, electrooculográficas, electromiográ- ficas, electrocardiográficas, etc...., han permitido establecer un paralelo entre la profundidad del sueño y esos índices electrofisiológicos (Loomis y cols., 1937). Este paralelismo se ve limitado "paradójicamente» cuando, en un momento dado, a las ondas delta asociadas al sueño profundo (el dormir clásico) sucedía una fase de sueño aún más profundo en que la mayoría de los indicadores electrofisiológicos son similares a los de la vigilia activa: desincronización del EEG, reaparición de movimientos oculares, indicadores que a su vez se asocian "paradójicamente», a una importante atonía muscular (Jouvet y cols., 1959; Dillon y Webb, 1965).

Aserinski y Kleitman demostraron que esta forma de sueño aparece cíclicamente a lo largo de la noche. Se asocia con la actividad onírica (Dement y Kleitman, 1957) y es una fase en la que la excitabilidad cortical es tan importante como durante la vigilia, por lo que ha sido denominada «paradójica» por Michel Jouvet y sus colaboradores de la escuela de Lyon (Jouvet y cols., 1959). También se le han puesto otros nombres: sueño desincronizado o sueño $\ll \mathrm{D}$ » (Hatman, 1975) y sueño REM (Rapid Eve Movement). La mayor parte de los autores consideran que esta fase constituye un tercer estadio del cerebro (Snyder, 1963; Dement, 1965).

Si existen dos estados diferentes de sueño, es posible que realicen funciones diferentes: esta hipótesis de trabajo ha sido muy fructuosa desde el principio de los años 60. Los psicofisiólogos del mundo entero se han interesado por el sueño paradójico (SP): La mayoría de las hipótesis formuladas relacionaban, lógicamente, sueño paradójico y actividades cognitivas; la hipótesis inicial de Jackson se precisaba. En efecto, ya que todas las actividades cognitivas necesitan una cierta activación del SNC, el SP, un sueño 
«activo», era el mejor candidato como soporte de la cognición.

Desde el punto de vista electrofisiológico, el SP se acerca mucho a la vigilia. En realidad es esto lo que le ha valido este nombre: el EEG es desincronizado y de bajo voltaje, el EOG testifica movimientos oculares rápidos, por el contrario, el EMG es uniforme, es decir, que la atonía muscular es máxima.

La presencia simultánea de la desincronización del EEG, de movimientos oculares rápidos y de atonía muscular permite el diagnóstico de sueño paradóiico (Rechstaffem y Kales, 1968).

Otras varias características señalan y confirman el SP: aparición de ondas Theta hipocámpicas (Cadilhac y Cols, 1961; Weis y Roldan, 1964), descargas ponto-geniculo-occipitales, descargas PGO (Brooks y Bizzi, 1963; - Dement y Cols, 1969; Dement, 1970), movimientos rápidos de otros músculos así como modificaciones de fenómenos vegetativos.

Moruzzi (1965), ha reagrupado estas diferentes características en componentes tónicos y componentes fásicos. Llama «componentes tónicos» a la caída del tono muscular, la desincronización cortical, las ondas theta hipocámpicas, que se mantienen durante todo el período de sueño paradójico. Llama «fásicos» a los componentes que aparecen de manera discontínua por salvas, en el curso del SP: éstos son esencialmente los movimientos oculares rápidos, los puntos PGO, los movimientos rápidos de otros músculos y los fenómenos vegetativos.

El paso del sueño clásico al sueño pa'radójico, y viceversa, no es inmediato y brutal. Entre el sueño lento y el sueño paradójico existe un corto período de transición durante el cual coexisten índices de sueño lento y de sueño paradójico: EEG desincronizado de bajo voltaje con presencia de ondas Delta de voltaje más elevado y de complejos «K», presencia de algunos movimientos oculares rápidos, pérdidas breves del tono muscular. Lairy $(1966,1967)$ ha distinguido este período de transición que encuadra el período de SP y le ha denominado fase intermedia (PI) de sueño. Esta fase intermedia puede ser considerada como la fase de arranque del SP.

\section{SUENOO PARADOJICO Y ACTIVIDADES COGNITIVAS}

Las funciones del sueño paradójico han sugerido numerosas hipótesis y han dado lugar a numerosos trabajos. Hennevin y Laconte (1971) han hecho una revisión completa de ellos.

Entre esas numerosas funciones es necesario retener la función determinante del sueño paradójico en las actividades cognitivas.

Durante el último decenio han nacido casi simultáneamente, dos tipos de trabajos complementarios, ricos en resultados, y que abren perspectivas nuevas. Estos trabajos relacionan, por una parte, el sueño paradójico y el aprendizaje, y por otra, el sueño paradójico con la inteligencia.

El papel del sueño en la retención es un hecho de experiencia común y estudiado desde hace tiempo por los psicólogos experimentales (Jenkins y Dallenbach, 1924).

El descubrimiento del sueño paradójico, de sus relaciones con la memoria y el aprendizaje, ha reforzado la hipótesis de un mecanismo activo de consolidación, de orden bioquímico sin duda alguna.

El papel del sP en la memoria y en el aprendizaje se ha probado tanto en el hombre como en el animal por medio de metodologías diversas. Los trabajos franceses más recientes, los de Laconte 
(1975) y de Dubais-Hennevin (1976), llegan a la conclusión inequívoca de que el sueño paradójico tiene una función determinante en el proceso de memorización. Durante esa fase particular, se efectuarían las diferentes síntesis que permiten la integración y el almacenaje de la información.

Entre los trabajos sobre las funciones del SP ocupan un lugar aparte los que se interesan por las relaciones entre $S P$ e inteligencia. Se refieren a un amplio dominio de la psicología, definido de manera diferente según los autores. Además en este dominio de investigación, solamente se puede utilizar el método patológico, al menos en el hombre. El SP en esta metodología se considera obligatoriamente como variable independiente, que sólo podrá relacionarse con otra variable independiente, en concreto la debilidad mental. Las relaciones entre estas dos variables sólo podrán expresarse en términos de correlación; las relaciones de causalidad, por el momento, sólo podrán ser hipotéticas.

Petre-Quadens (1966) fue la primera que estudió el sueño paradójico de los débiles mentales. Su primer estudio se realizó sobre 43 débiles mentales patológicos (según la terminología propuesta por Chiva [1969/1973]) y 28 sujetos normales. El abanico de edad de los débiles mentales iba de tres meses a 36 años. Las diferencias entre débiles mentales y normales exan significativas en lo que concierne al SP, tanto desde el punto de vista de los componentes tónicos como de los componentes fásicos. Petre-Quadens observó una reducción significativa $(\mathrm{P}<.001)$ de la tasa de sueño paradójico en los débiles mentales. $\mathrm{La}$ tasa media de sP de los débiles mentales era del 15,6 por 100, la de los normales 25,9 por 100. Observó además una disminución de la cantidad de movimientos oculares de las fases de SP de los débiles mentales. Sus trabajos posteriores confirmaron sus observaciones primeras. Además (1969) comprobó que la disminución de la tasa de SP con la edad (Roffward y cols., 1966) es más acusada en los débiles mentales.

Un trabajo análogo realizado con 150 débiles mentales patológicos de 0 a 62 años, llegó a las mismas conclusiones (1972).

El análisis de las grabaciones de los sueños nocturnos de los débiles mentales, condujo a Petre-Quandens a distinguir un nuevo tipo de sueño, el sueño indeterminado, cuyas características son una actividad EEG de bajo voltaje con la presencia de ondas en diente de sierra, atonía muscular, sin movimientos oculares rápidos. Parece existir una relación inversa entre la tasa de SP y la tasa de sueño indeterminado.

Desde 1969, Petre-Quadens, se ha interesado especialmente por el estudio de los movimientos oculares rápidos, más en concreto por los intervalos entre los movimientos oculares consecutivos de las fases paradójicas. Son esencialmente los intervalos cortos (inferiores a $1 \mathrm{sg}$ ) los que son deficitarios en los débiles mentales, tanto los movimientos oculares verticales como los movimientos oculares horizontales. El porcentaje medio de intervalos inferiores a 1 segundo se correlacionaba positivamente con el $\mathrm{cr}$ : era de 1,65 por 100 en tres sujetos con cr inferior a 30, de 8,68 por 100 en tres sujetos con CI de 60 , y de 24,33 por 100 en dos sujetos con CI superior a 125 . Petre-Quadens considera que los períodos de intensidad débil de movimientos oculares, son períodos de inhibición cortical.

Petre-Quadens estima que sus resultados tienden a confirmar la hipótesis «sleep-cognition». Admite, además, que todas las formas de debilidad mental están asociadas a un déficit de monoaminas 
y que la disminución de la tasa de SP en los débiles mentales no es más que un efecto de este déficit. Esta seductora hipótesis, que se apoya sobre todos los trabajos que conciernen la bioquímica del SP, trabajos de la escuela de Lyon, en particular (Jouvet y Pujol, 1972; Mouret y cols., 1972; Jouvet, 1974), ha sido objeto de un principio de verificación (Petre-Quadens y De Greef, 1971). La administración de 5-Hidroxytryptamina ( 5 HTP), precursor de la serotonina ( $5 \mathrm{HT}$ ) a cuatro mongólicos de cuatro semanas, dos meses, diez meses y cuatro años y medio, ha producido un aumento significativo de la cantidad de movimientos oculares en su SP, así como del número de intervalos inferiores a 1 sg que se aproxima al de los niños normales. Este trabajo no aporta, desgraciadamente, ninguna conclusión en lo que concierne a una posible mejoría de las posibilidades psicomotrices e intelectuales de los sujetos tratados, pero permite especulaciones optimistas.

Feinberg y sus colaboradores (1967) habían observado una reducción importante de la tasa de SP en los sujetos aquejados de síndrome cerebral crónico (chronic brain syndrom). Con el fin de apoyar más su interpretación, que relaciona el déficit intelectual causado por el síndrome cerebral crónico y la reducción de la tasa de SP, generalizaron su hipótesis a los débiles mentales.

Este nuevo trabajo de Feinberg y de sus colaboradores (1969) apunta a mostrar la generalidad de relaciones entre SP e inteligencia para todos los tipos de déficits intelectuales.

La población estudiada se compone de sujetos adultos: 21 débiles mentales patológicos, 11 indiferenciados (débiles mentales normales y de etiología desconocida), 15 sujetos normales así como 13 ancianos aquejados de síndrome cerebral crónico. Utilizaron como Petre-
Quadens, el sistema de clasificación de los estadios del sueño de Dement y Kleitman (1957). Los débiles patológicos tienen una tasa de SP significativamente inferior a la de los sujetos normales; la diferencia no es significativa para los indiferenciados.

Los autores obtuvieron un coeficiente de correlación de .74 entre la tasa de SP y los resultados de la escala verbal del wPPSI y de .64 con la escala manipulativa. Estos dos coeficientes son significativos a $p<.001$. La de Spearman entre la. tasa de SP y las puntuaciones en el Binet-Simon era .77. Feinberg y sus colaboradores comprobaron que la correlación media entre la tasa de SP y las puntuaciones en la escala completa del wPPSI .73 , era independiente de la edad.

Consideran que se verifica la hipótesis «sleep-cognition» pero afirman que no hay nexo de causalidad directa entre las alteraciones del cerebro que deterioran los mecanismos del sueño y los que afectan a los de la inteligencia. Sin embargo, no rehusan, a priori, una interpretación en términos de causalidad directa que haría intervenir procesos de crecimiento sináptico.

Los trabajos de Castaldo (1968) aportan resultados análogos. Un estudio realizado con 16 adolescentes mongólicos, muestra que las tasas más débiles de $\mathbf{S P}$ se asocian a los mayores déficits intelectuales. Castaldo y Krynicki estudian 'el sueño de los débiles mentales «funcionales» para mostrar la generalidad de la relación inteligencia SP. Estos débiles mentales funcionales no presentan ninguna lesión orgánica; se pueden considerar como débiles mentales normales. Con siete sujetos de 13 a 16 años, los autores observaron una tasa media de SP de 17,49 por 100 . La correlación entre la tasa de SP y el cr, estimada en Stanford-Binet, era de .723 (significativo a $\mathrm{p}<.10$ ). La correlación entre la tasa de 
SP y el coeficiente social estimado con la escala de madurez social de Vineland era de .710 (significativa a $\mathrm{p}<.10$ ). Los autores observaron que la tasa de sp correlacionaba negativamente con la latencia de la primera fase del SP.

Estos resultados se confirman en un estudio realizado con seis mongólicos y seis débiles funcionales (Castaldo y Krynicki). Los autores comprobaron que la cantidad de movimientos oculares de las fases de SP, correlacionaba positivamente con los CI (.673, significativo a $\mathrm{p}<.05$ ).

Los resultados obtenidos por Castaldo y Krynicki se interpretaron a la luz de la hipótesis de que la relación entre el SP y la inteligencia de los débiles mentales no tiene su origen en una etiología orgánica sino que depende de una relación general. Estos autores interpretan esta relación a la luz de la hipótesis de Dewan (1969, 1970). Dewan estima que una de las funciones del SP es la integración de la información. La debilidad mental se asociaría con una integración deficitaria debida a una falta de sueño paradójico.

Esta interpretación en términos de causalidad ha conducido a Castaldo y a sus colaboradores (1973) a pensar que toda mejoría de la cantidad de SP sería susceptible de aportar una mejoría de los rendimientos intelectuales. La L-DOPA, que mejora secundariamente las posibilidades intelectuales de los parkinsonianos (Maier y Martin, 1970), ha sido utilizada en 14 sujetos normales y dos débiles mentales. No se ha probado ninguna acción significativa, ni sobre la tasa de SP ni sobre las puntuaciones intelectuales.

Schmidt y sus colaboradores, en un estudio de 1968 , comprobaron que la cantidad media de SP de los adolescentes mongólicos es significativamente inferior a la de los adolescentes normales: $78 \mathrm{mn}$ VS 90,4 mn. Schmidt y Kaelbling (1972) confirmaron estos resultados: la duración y el número de fases del sP son significativamente inferiores en los débiles mentales.

Un estudio reciente de Clausen y sus colaboradores (1977) realizado con 21 mongólicos y 21 sujetos normales de ambos sexos, confirmó los resultados obtenidos en 1974 por Fukuma y cols. La duración del SP es significativamente superior en los normales, la tasa de sueño indeterminado es superior en los mongólicos, la latencia de la primera fase de SP se alarga en los mongólicos, además, el

Cundro 1.-Indices comparativos de los sueños de sujetos normales y deficientes mentales según Grubar, 1977

\begin{tabular}{l|c|c|c|c}
\hline & $\begin{array}{c}\text { Deficientes } \\
\text { patológicos }\end{array}$ & $\begin{array}{c}\text { Deficientes } \\
\text { normales }\end{array}$ & $\begin{array}{c}\text { Deficientes } \\
\text { indeterminados }\end{array}$ & $\begin{array}{c}\text { Sujetos } \\
\text { normales }\end{array}$ \\
\hline N & 15 & 7 & 2 & 8 \\
\% SI & 12,14 & 16,28 & 15,80 & 72 \\
$\%$ SP & 4,42 & 3,09 & 3,18 & 1,09 \\
Lat. & 122,60 & 111,80 & 118,50 & 99 \\
Fase SP & 26,06 & 36,28 & 29,50 & 175,37 \\
(mn) & 36,73 & 37,85 & 36,50 & 161,12 \\
Int 1 s & 11,40 & 8,28 & 8 & 8,50 \\
Int 1 S & 27 Fases & 65 & 53,5 & 102,5 \\
SI & & & & \\
OI & &
\end{tabular}


cr correlaciona con la cantidad de movimientos oculares rápidos en los mongólicos.

Trabajando con sujetos deficientes mentales de diversas etiologías, Grubar (1973, 1975, 1976, 1977), a partir de grabaciones nocturnas analizadas según la clasificación propuesta por Rechtsaffeu y Kales, 1968), obtuvo resultados coincidentes con los presentados anteriormente.

Un estudio de 1977, realizado con 24 débiles mentales ( 15 patológicos, siete normales, dos indeterminados) y ocho sujetos normales de la misma edad cronológica, permitió comprobar que existe una relación entre el cI, estimado con el wisc, y la tasa de SP: el coeficiente de correlación ordinal ( $\rho$ de Spearman) obtenido a partir de estos dos parámetros es de .85 (significativo a $\mathrm{p}<.01$ ). La tasa media de SP de los débiles mentales (comprendidas todas las etiologías) es significativamente inferior a la de los normales (13,75 por 100 versus 22 por 100 , significativo a $\mathrm{p}<.01$ ).

La latencia de la primera fase de sP es significativamente mayor en los débiles mentales ( $119 \mathrm{mn}$ vs $99 \mathrm{mn}$, significativo a $\mathrm{p}<.01$ ).

La tasa media de sueño intermediario (definido por Lairy, 1966), es significativamente superior en los débiles mentales $(3,68$ por 100 vs 0,96 por 100 , significativo a $\mathrm{p}<.01$ ), lo mismo que el número de fases de este tipo de sueño $(10,20$ vs 8,5 , significativo a $\mathrm{p}<.05)$. Este alargamiento de las fases de sueño intermediario se muestra, sobre todo, al principio del sueño. Este resultado coincide con los de Lairy (1966), que comprobó que la duración de estas fases de sueño tiene una tendencia a aumentar en los débiles mentales, así como con los de Goldie y cols., 1968, quienes habían observado, en cinco mongólicos recién nacidos, un aumento de los períodos de transición entre sueño lento y sueño paradójico. El aumento del número de fases de sueño intermediario puede interpretarse como un índice de «aborto» del sueño paradójico en los débiles mentales, cuyo origen es, sin duda, de orden bioquímico.

Además, los intervalos cortos entre movimientos oculares rápidos son significativamente inferiores en los débiles mentales: con ello vuelven a confirmarse los resultados de Petre-Quadens (1969, 1972).

Parece que las variables analizadas por Grubar (tipo etiológico, CI, tasa de SP, tasa de sueño intermediario, intervalos entre movimientos oculares rápidos) son interdependientes (ver cuadro 1).

Estos resultados apoyan la hipótesis de la generalidad de la relación entre inteligencia y tasa de sueño paradójico, formulada ya por Castaldo, cualquiera que sea el tipo etiológico considerado. El déficit del sueño paradójico de los débiles mentales no tiene necesariamente un origen lesional, sino que es una característica del síndrome de deficiencia mental.

\section{INTERPRETACION Y CONCLUSIONES}

Existe una relación entre la deficiencia mental y la tasa de sueño paradójico, más exactamente entre los niveles intelectuales inferiores o medios y la tasa de SP.

Podemos suponer que esta relación puede generalizarse a todos los niveles intelectuales: los superdotados (CI superior a 140) tendrían una tasa de SP superior a la de los normales. Esta relación deficiencia mental-sueño paradójico sería en realidad, una manifestación de una relación más general entre inteligencia y tasa de SP (hipótesis 1). 
Esta relación se observa en los niveles intelectuales inferiores, cualquiera que sea la etiología del déficit intelectual.

La mayoría de las investigaciones emprendidas en este dominio tenían por objeto la verificación de la hipótesis de Jackson que relaciona sueño y actividades cognitivas.

Los resultados obtenidos por diversos autores, desde 1966, son unánimes y favorables a la hipótesis jacksoniana y son convergentes con los que establecen una relación entre la memoria y el sueño paradójico.

Sin embargo aún no se ha propuesto ninguna interpretación satisfactoria de los mecanismos en juego en esta relación.

Petre-Quadens (1966) propone un determinismo de orden monoaminérgico, determinismo que eventualmente sería directo. Castaldo y Krynicki (1973) apoyándose en la hipótesis de Dewan (1970), orientan su interpretación en el sentido de un lazo de causalidad directa entre el SP y la deficiencia mental. La interpretación de Grubar (1977) se aproxima a la de Castaldo, pero hace intervenir la memorización como variable intermediaria entre el SP y la debilidad mental.

Todas estas interpretaciones son todavía hipotéticas y no definen de forma completamente satisfactoria los mecanismos en juego y las vías de determinación implicadas.

Hay que subrayar que la utilización del término «relación» entre el sueño paradójico y el nivel intelectual ya deja la puerta abierta a una interpretación implícita. Quien dice «relación», dice «nexo» $y$, por tanto, dependencia de un parámetro con relación al otro. Esta interpretación implícita se refuerza por la utilización, en la mayoría de los autores, del coeficiente de correlación. La tasa de SP y el nivel intelectual correlacionan positivamente. Como el coeficiente de correlación es una media de «no-independencia» entre dos variables, la posibilidad de independencia entre la tasa de SP y el CI se rechaza a priori.

Sin embargo, la «correlación» no implica necesariamente «nexo causal», no prejuzga acerca del origen de la causalidad.

La simple constatación de una correlación entre la tasa de SP y el nivel intelectual es, de por sí, una información interesante. Esta constatación, en caso de verificarse para los niveles intelectuales superiores, permitiría considerar la tasa de SP como un índice del nivel intelectual, como una medida objetiva de la inteligencia, una medida directa y no mediatizada por instrumentos más o menos teñidos culturalmente. Los trabajos de Roffwarg y sus colaboradores (1966) sobre la autogénesis del SP han proporcionado lo que podría constituir un marco de referencia.

Para que la tasa de sueño paradójico pueda constituir efectivamente un índice del déficit intelectual, es necesario que el carácter deficitario de la tasa se mantenga constante; cualquiera que sea la edad cronológica de los individuos, la curva de disminución ontogenética del sP de los deficientes mentales deberá permanecer constantemente inferior a la de los normales. Esta segunda hipótesis está verificándose actualmente (cuadro 2 y figura 1).

Puesto que el sueño paradójico puede registrarse y diferenciarse desde el nacimiento (Petre-Quadens, 1966), su empleo puede ser muy precoz con todas las ventajas consiguientes para un diagnóstico precoz de la deficiencia mental.

El empleo de instrumentos sofisticados (polígrafo) y la duración de la investigación del sueño, constituyen los únicos obstáculos para la utilización de esta técnica en el diagnóstico de la deficiencia mental. 
Cundro 2.-Indice de sueño paradófico en función de la edad. Deficientes mentales $y$ normales. $1 / 1978$

\begin{tabular}{c|c|c|c|c}
\hline \multirow{2}{*}{$\begin{array}{c}\text { Edad } \\
\text { (años) }\end{array}$} & \multicolumn{2}{|c|}{ Deficientes mentales } & \multicolumn{2}{c}{ Normales } \\
\cline { 2 - 5 } & N..$^{\circ}$ sujetos & $\%$ sP & N. sujetos & $\%$ sP \\
\hline $5-6$ & 1 & 17,50 & 0 & - \\
$6-7$ & 3 & 15,51 & 1 & 22,45 \\
$7-8$ & 2 & 14,54 & 2 & 22,50 \\
$8-9$ & 2 & 9,68 & 2 & 22,32 \\
$9-10$ & 1 & 14,30 & 1 & 18,72 \\
$10-11$ & 5 & 14,20 & 0 & - \\
$11-12$ & 3 & 13,04 & 1 & 21,34 \\
$12-13$ & 7 & 12,79 & 2 & - \\
$13-14$ & 2 & 13,74 & 0 & - \\
$14-15$ & 1 & 9,60 & 0 & - \\
$15-16$ & 1 & 11,55 & 0 & - \\
\hline
\end{tabular}

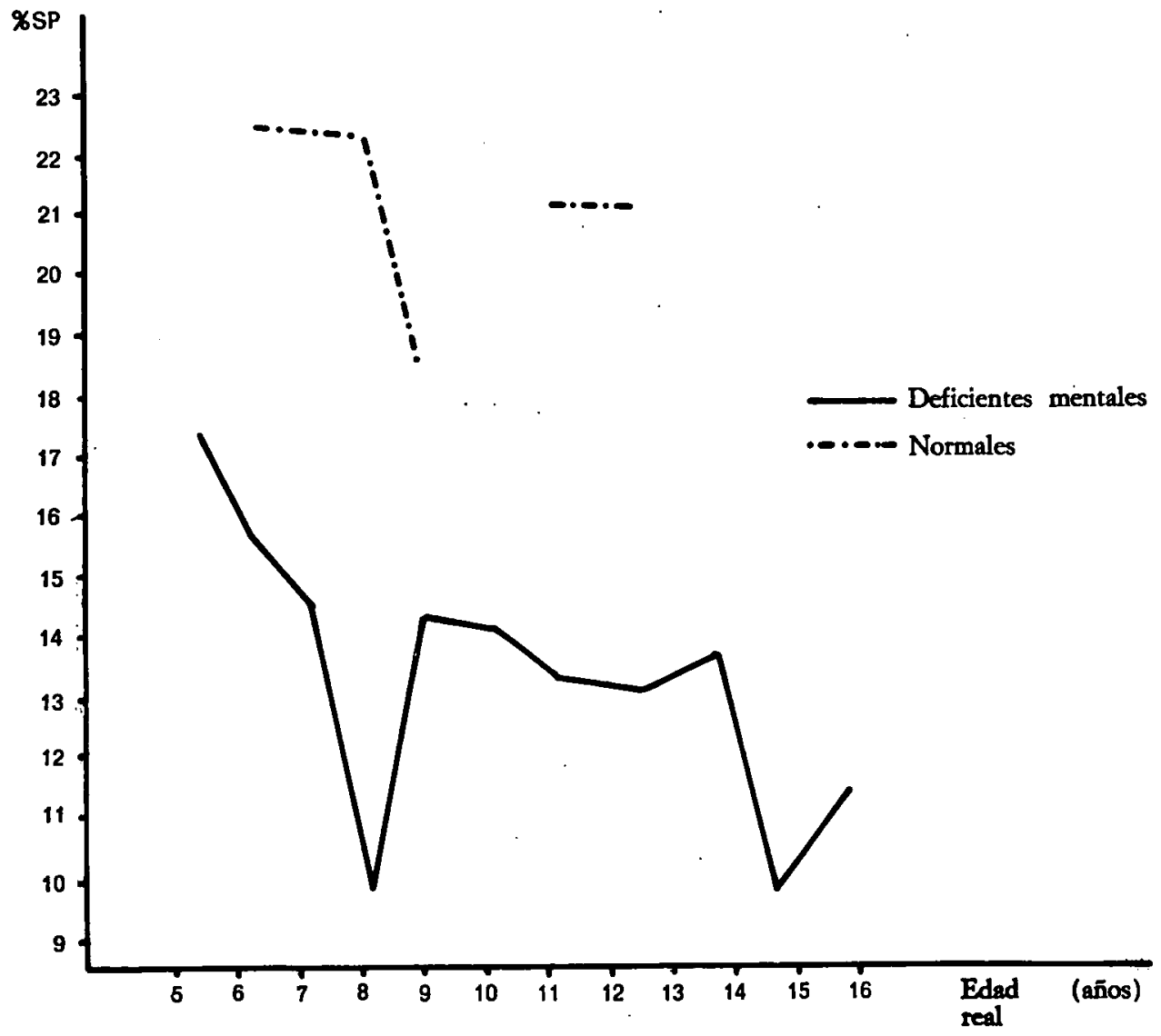

Figura 1.-Variaciones del indice de SP de deficientes mentales y de normales en función de la edad. Grubar, 1978 
Además esta técnica podría utilizarse para el diagnóstico diferencial entre la deficiencia mental y el autismo (Roelandt, 1975).

Esta interpretación se basa en una concepción innatista del SP que relaciona la tasa de SP y la maduración (JouvetMounier, 1968). El sueño paradójico demuestra la plasticidad del sistema nervioso (Jouvet, 1972). En el curso del SP tendría lugar la repetición de conductas innatas programadas genéticamente (Jouvet, 1974). A la luz de este enfoque, el deficiente mental tendría un sistema nervioso madurado precozmente $y$ dispondría desde un principio de menos programas innatos.

Si se define la inteligencia como la aptitud para aprender, la interpretación innatista explica perfectamente los déficits específicos de aprendizaje de los deficientes mentales. Esta interpretación es muy coherente con los trabajos de Piaget que deriva la inteligencia de los esquemas innatos (1936 y 1967 fundamentalmente) y los trabajos de Inhelder (1959). El nexo entre memoria e inteligencia ha sido, además, objeto de un libro (1968) en el que Piaget e Inhelder, confirman sus posiciones. Esta interpretación es coherente también con la teoría selectiva del aprendizaje propuesta por Changeux.y Danchin (1974).

A este tipo de interpretaciones estáticas, hay que añadir los trabajos sobre la filogénesis de los primates: los trabajos de Gastaut y Bert (1974) y la interpretación estructural de Oswald demuestran un nexo entre el número de cromosomas, la inteligencia y el coeficiente de sueño paradójico.

Este enfoque interpretativo no señala una causa a priori sino que intenta, simplemente, arrojar alguna luz sobre la deficiencia mental.

Por el contrario, la mayoría de los tra- bajos que demuestran la dependencia (por lo menos una cierta dependencia) de la tasa de sP con respecto al entorno, desembocan en interpretaciones causales, acerca de la relación entre la tasa de SP y la deficiencia mental.

Si la tasa de SP varía con la maduración, depende también de variables momentáneas: ciertas condiciones del entorno (externo o interno) son súsceptibles de aumentarla.

Las situaciones de aprendizaje implican un aumento de la tasa de sP consecutivo; esta afirmación se ha verificado en los deficientes mentales (Castaldo, 1972). La administración de 5-HTP a mongólicos se sigue de un aumento de la tasa de SP (Petre-Quadens y De Greef, 1971). Es una pena que no se aporte ningún dato sobre una mejoría eventual, debida a esas manipulaciones, de los resultados y posibilidades intelectuales. Podemos suponer que el aumento de la tasa de SP, consecutivo a diversas clases de manipulaciones del entorno, será menor en los deficientes mentales que en los normales.

Esos resultados son fácilmente interpretables a partir de la hipótesis «P» de Dewan (1969). Justamente durante las fases de SP tendría lugar la puesta a punto de los nuevos programas que soportarían los aprendizajes nuevos, de ahí el aumento de la tasa de SP consecutivo a los procesos de memorización y, a la inversa, podemos suponer que todo aumento artificial del sp implica la posibilidad de elaborar más programas nuevos; cualquier aumento del SP consecutivo implicará una facilitación de los aprendizajes. Esta hipótesis se apoya en la siguiente cadena de determinación:

$$
\text { sP } \rightarrow \text { Memoria a largo plazo }
$$

y ha sido verificada en animales por Delacour y Brenor (1975). Las implicaciones pedagógicas de esta hipótesis son 
importantes, por lo que debería experimentarse en el hombre.

Con ello se vuelve a plantear la hipótesis de que hay una cadena de determinación que enlaza al sueño paradójico con la memoria a largo plazo y la memoria a largo plazo con la inteligencia (hipótesis 3).

Todo ello plantea el problema de las relaciones entre el aprendizaje y la inteligencia, entre la memoria y la inteligencia. Los autores relacionan, cada vez más, aprendizaje, memoria e inteligencia (de Montpellier, 1976). Para la escuela piagetiana, existe una continuidad entre memoria e inteligencia; esta continuidad se ha evidenciado también en las investigaciones neuroquirúrgicas y especialmente en los trabajos de Milner (1969). $\mathrm{La}$ inteligencia procede de lo que ha sido memorizado por la especie o por el individuo.

En esta perspectiva precisamente se sitúa la teoría de los dos niveles de apti. tudes intelectuales de Jensen (1970). El nivel «I» que corresponde a la memoria a corto plazo, es anterior a la aparición del nivel «II», la abstracción. A la luz de la teoría de Jensen, la deficiencia mental puede definirse como la incapacidad para el paso del nivel «I» al nive] «II» cuyo origen podría explicarse bien porque el nivel «I» es deficitario (insuficiencias de adquisiciones a corto plazo), interpretación que estaría en concordancia con Ellis (1963), o bien porque hay utilización deficitaria de esas adquisiciones. Se denomina primaria a la deficiencia mental con origen en el nivel «I» (que frecuentemente es muy profunda), mientras que aquella cuyo origen está en el nivel II, se denomina secundaria y es generalmente ligera.

Este modelo explicativo da cuenta precisa de la correlación entre tasa de SP e inteligencia: cuanto más capaz es el individuo de procesar un gran número de informaciones, es decir, cuanto más elevado es su índice de SP, tanto más inteligente es. Esta hipótesis es coherente con los trabajos que muestran los déficits mnemónicos de los deficientes mentales (Borelli-Vincent y cols., 1962; Roodin y cols., 1976, especialmente).

En esta orientación explicativa, la tasa de sP juega el papel de variable independiente. Es imposible no tener en cuenta los trabajos que han tratado el índice de SP como variable dependiente, es decir, que consideran que el SP está determinado por un antecedente constituido especialmente por la cantidad de informaciones a procesar, determinada a su vez por la cantidad de informaciones almacenadas a corto plazo.

Numerosos estudios en particular los de Ellis (1963 y 1970), de Simson y Wetherick (1973) han confirmado la existencia de un déficit de la memoria a corto plazo en los débiles mentales. Este déficit de Almacenaje a corto plazo es capaz de acarrear una necesidad menor de SP. En esta perspectiva, el déficit de SP no es primario sino secundario; la relación entre tasa de SP y debilidad mental puede explicarse por la siguiente secuencia determinista:

$\mathrm{MCP} \rightarrow \mathrm{SP} \rightarrow \mathrm{MLP} \rightarrow \mathrm{DM} \quad$ (bipótesis 4).

Sin embargo, ya que actualmente no se dispone de ningún trabajo sobre las relaciones entre memoria a corto plazo y sueño paradójico, es prudente considerar otro esquema determinista, esquema que asocia simultáneamente un déficit de la memoria a corto plazo y un déficit del SP, independientes uno del otro:

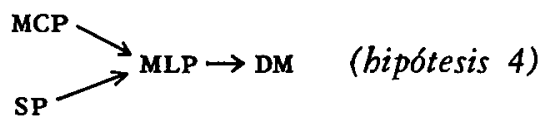

En este esquema, el déficit en SP refuerza el déficit de la memoria a corto plazo. 
Es posible postular, siguiendo la teoría de los dos niveles, que existen debilidades mentales con y sin déficits de la memoria a corto plazo; las debilidades mentales profundas serían debilidades mentales primarias cuyo origen sería un déficit simultáneo de la memoria a corto plazo y del sp; las deficiencias mentales medias y ligeras serán deficiencias mentales secundarias en las cuales no existiría déficit de la memoria a corto plazo sino simplemente un déficit del SP (eventualmente ligero).

Establecer un paralelismo entre las distinciones de Jensen y de Chiva puede ser muy interesante: los deficientes mentales patológicos no serían sino deficientes mentales primarios, los deficientes mentales normales, deficientes mentales secundarios (hipótesis 5). Los deficientes mentales patológicos cuyo origen es por lesión se asociarían a déficits de la memoria a corto plazo y a déficits del SP susceptibles de tener un origen de lesión. Las deficiencias mentales normales que, por definición no son causadas por una lesión, no estarían asociadas a un déficit de los mecanismos de la memoria a corto plazo, sino asociadas a un déficit del SP. (1)

Los diversos sistemas explicativos presentados hasta el momento, se sitúan en una dimensión etiológica: todos ellos consideran que la deficiencia mental no es más que una consecuencia de déficits anteriores (MCP, MLP y SP).

Podemos considerar, y ello constituye el otro polo de la alternativa, que la deficiencia mental es la causa de déficits de la MCP, de la MLP, del sP, lo mismo que es posible pensar que la deficiencia $y$ otros déficits tienen su origen en una alteración del sistema nervioso central, hipótesis muy probable en el caso de las deficiencias mentales patológicas.
En el actual estado de saber, no es posible todavía determinar cuál es el origen efectivo de la cadena de determinismos. Numerosos trabajos apuntan la necesidad de verificar las cinco hipótesis presentadas, que son indispensables:

- Estudio del índice del sp de los superdotados (hipótesis 1).

- Disminución ontogenética del índice de sp de los deficientes mentales y de los normales (hipótesis 2-trabajo en curso).

- Estudio de las consecuencias de todo aumento del índice de SP sobre los resultados intelectuales (hipótesis 3).

- Estudio de las relaciones entre memoria a corto plazo y SP (hipótesis 4).

- Estudio de los déficits de la memoria a corto plazo de los deficientes mentales normales y patológicos (hipótesis 5-trabajo en curso).

Los otros índices fisiológicos evocados al principio de este estudio, son también analizados en términos de correlación. Ningún sistema explicativo es suficiente para dar cuenta de los nexos que existen entre EEG e inteligencia, potenciales evocados e inteligencia.

Sólo un enfoque pluridisciplinar: bioquímico, fisiológico y psicológico de la deficiencia mental y del sueño paradójico podría explicar la correlación índice de sp-deficiencia mental, y eventualmente, las bases orgánicas de la inteligencia.

En espera de que las hipótesis formuladas sean verificadas, es conveniente continuar considerando la tasa de SP solamente como un índice de los niveles medios e inferiores de la inteligencia. 


\section{Resumen}

La investigación de indices fisiológicos correlativos de la eficiencia intelectual, ba sido la preocupación constante de numerosos psicofisiólogos.

El análisis del EEG, el análisis de los potenciales evocados de niños defifientes mentales $y$ de niños normales conducen a resultados contradictorios.

El descubrimiento del sueño paradójico ba sido el origen de un nuevo enfoque de la eficiencia intelectual. Numerosos trabajos con resultados convergentes, muestran una correlación positiva entre tasa de sueño paradófico y deficiencia mental.

¿Puede generalizarse esta relación a todos los tipos etiológicos y a todos los niveles intelectuales? ¿Qué mecanismos entran en juego? Se propone una interpretación que bace intervenir la memoria como variable intermediaria.

\section{Résumé}

La recherche d'indices physiologiques corrélatifs de l'efficience intellectuelle, a été le souci constant de nombreux psychopbysiologistes.

L'analyse de l'EEG, l'analyse des potentiels évoqués d'enfants déficients mentaux et d'enfants normaux n'ont amené que des résultats contradictoires.

La découverte du sommeil paradoxal a été a l'origine d'une nouvelle approcbe de l'efficience intellectuelle. De nombreux travaux dont tous les résultats convergent, font ressortir une corrélation positive entre taux de sommeil paradoxal et déficience mentale.

Cette liaison peut-elle être généralisée à tous les types étiologiques et pour tous les niveaux intellectuels? Quels sont les mécanismes en jeu? Une interprétation faisant intervenir la mémoire, comme variable intermédiaire est proposée.

\section{Summary}

The investigation of physiological correlates of mental efficiency bas been the constant worry of many psychopbysiologists.

Tbe EEG and the evoked potentials analysis of retarded and normal children lead to inconsistent results.

The discovery of REM sleep originated a new approach of mental efficiency. Many works with consistent results sbow a correlation between REM sleep rate and mental deficiency.

Can this relationship be generalized to every etiological type and to every intellectual level? Which mechanisms are concerned? An interpretation with memory as an intermediate parameter is proposed.

\section{Nota}

(1) Los trabajos de Moinet y Behague (1978) sobre la reproducción de estructuras rítmicas verifican la primera parte de la hipótesis: los deficientes mentales patológicos presentan déficits de la MCP más acentuados que los deficientes mentales normales. 


\section{Referencias}

ASERINSKi, E. y KLeITMAN, N., «Regularly occuring periods of eye motility and concomitant phenomena during sleep», Science, 1953, 118, 273-274.

BASER, A. B. y LODGE, A., «Click evoked EEg responses in normal and developmentally retarded infants», Nature, 1967, 214, 252-255.

Berger, H., «Ueber des Elektrenkephalogram des Menschen», Archiv Psychiatry, 1929, 87, 527.570.

Blume, W. T., David, R. B. y Gómez, M. L., «Generalized sharp and slow waves complexes: associated clinical features and long term follow up», Brain, 1973, 96, 289-306.

Borelli-Vincent, M., Hurtig, M. y Perron, R., «Memorisation, niveau mental et niveau scolaire», Enfance, 1962, 4/5, 397-431.

Brooxs, D. y Bizzi, E., «Brain stemelectrical activity during sleep», Archiva Italiana Psychiatria, $1963,101,648-665$.

Cadilhac, J., Passount-Fontaine, T. y Passouant, P., «L'organisation des divers stades du sommeil chez le chaton, de la naissance à 45 jours», Journal de Physiologie, 1961, 54, 305-306.

Callaway, E., «Correlation between average evoked potentials and intelligence tests", Process Symposium on Evoked Responses and Conditioning in Psychology and Psychiatry, 1973, INSERM, Tours.

CASTaldo, V., «EEG study of sleep patterns in adolescent mongoloïds», ASS. Psychophysiology Study Sleep, Denver, 1968.

CASTALDo, V., «Effects of a training program on the REM sleep of mentally retarded», Psychopbysiology, 1972, 9, 140.

CASTAldo, V. y KrinickI, V., «Sleep patterns and intelligence in functional mental retardation», Journal of Mental Deficiency Research, 1973, 17, 314, 231-235.

Castaldo, V. y Krinicki, V., «Sleep and eye movement patterns in two groups of retardates, Biological Psycbiatry, 1974, 9, 3, 231-243.

Castaldo, V., Krinicki, V. y Crade, M., «L DOPA and REM sleep in normal and mentally retarded children», Biological Psycbialry, 1973, 6, 295-299.

Chalke, F. C. A. y ERTL, J. P., «Evoked potentials and intelligence», Life Science, 1965, 4, 1319-1322.

Changeux, J. P. y Danchin, A., «Apprendre par stabilisation sélective de synapses en cours de développement», en L'Unité de l'Homme, Seuil, 1974, 320-350.

Chrva, M., Débiles normaux, débiles patbologiques, Delachaux et Niestlé, 1973 (versión cast. en Pablo del Río Editor, Madrid, 1977).

Chrva, M., «Tableaux psychologiques différentiels de la débilité mentale selon l'étiologie», en Les débilités mentales, A. Colin, 1969, 251-287.

Clausen, J., Sersen, E. A. y LidSkY, A., «Sleep patterns in mental retardation, Down's syndrome», Electroencephalography and ciinical neurophysiology, 1977, 43, 183-191.

Delacour, J. y BRenct, J., «Sleep patterns and avoidance conditioning in the rat», Pbysiology and Behavior, 1975, 14, 329-335.

Delorme, F., Jeannerod, J. y Jouver, M., «Effets remarquables de la réserpine sur l'activité EEG phasique ponto-gébiculo-occipitalew, Comptes Rendus de la Societé de Biologie, París, $1965,159,900$.

Dement, W., «Recent studies on the biological role of rapid eye movement sleep», American Journal of Psychiatry, 1965, 122, 404-407.

Dement, W., «The biological role of REM sleep (ca 1968)», en Sleep: physiology and pathology; a symposium, A. Kales (ed.), Lippincott, 1970, 245-265.

Dement, W. \& Kleitman, N., \&Cyclic variations in EEG during sleep and their relation to eye move ents, body motility and dreaming», Electroencephalography and clinical neurophysiology, 1957, 9, 673-690.

Dement, W., Zarcone, V., Ferguson, J., Cohen, H. y Pivix, T. y Barchas, J., «Some parallel findings in schizophrenic patients and serotonin depleted cats», en Schizophrenia current concepts and research, Siva Sankar (ed.) PID publications, 1969, 775-811.

Dewan, E., «The programming "P" hypothesis», Pbysical Sciences Paper, 1969, 388, 69-298.

DEwaN, E., «The programming (P) hypothesis for REM sleep», en Sleep and Dreaming, E. Hartman ed. Little, Brown and Co., 1970, 295.

Dillon, R. F. y WEBB, W. B., «Threshold of arousal from "activated" sleep in the rat", Journal of comparative Physiological Psychology, 1965, 59, 446-447.

Dubors-Hennevin, E., «Relations entre le sommeil paradoxal et les processus d'acquisition; approche des mécanismes en jeu», resis, París, 1976.

DuFFy, E., Activation and Bebavior, Wiley, 1962. 
Ellingson, R. J., «Relationship between EEg and test intelligence: a commentary», Psychological Bulletin, 1966, 65, 2, 91-98.

Ellis, N. R., "The stimulus trace and behavioral inadequacy», en Handbook of Mental Deficiency, N. R. Ellis (ed.), Mac Graw Hill, 1963.

Ellis, N. R., «Memory processes in retardates and normals», International Review of Research in Mental Retardation, 1970, 4, 1-32.

ERTL, J. P., «Evoked potentials and intelligence», Review of the University of Ottawa, 1966, $36,4,599-607$.

ERTL, J. P., "Evoked potentials, neurol efficiency and IQ», en Biocybernetics of the Central Nervous System, L \& D Proctor ed. Little and Brown, 1969.

ERTL, J. P. y SCHAFER, E. W., «Brain response correlates of psychometric intelligence», Nature (Londres), 1969, 223, 421-422.

Feinberg, I., Koresec, R. L. y Heller, N., «eeg Sleep patterns as a function of normal and pathological aging in man», Journal of Psychiatric Research, 1967, 5, 107-144.

Feinberg, I., Braun, M. y Schulman, E., «EEG sleep patterns in mental ratardation», Electroencepbalography and clinical neuropbysiology, 1969, 27, 128-141.

Fuxuma, E., Ukezawa, Y., Kobayashi, K. y Motorke, M., «Polygraphic study on the nocturnal sleep of children with Down's syndrome and endogenous mental retardation», Folia Psycbiatria Neurologia Japan, 1974, 28, 333-345.

GaSTAUT, H., «Correlations between the electroencephalographic and the psychometric variables (MMPI, Rosenzweig, intelligence tests)", Electroencephalography and clinical neurophysiology, $1960,12,226-227$.

GASTAUT, H. y BERT, J., «Primates, anthropoīdes et humains: les traits électroencéphalographiques communs», en L'unité de l'homme, Seuil, 1974, 174182.

Goldie, L., Curtis, J. P., Svendsen, U. y Roberton, N. R. C., «Abnormal sleep rhythms in mongol babies», Lancet, 1968, 1, 229-230.

Grubar, J. C., «Sommeil paradoxal et mongolisme», resultados inéditos, 1973.

Grubar, J. C., «Débilité mentale et sommeil paradoxal», Enfance, 1975, 3/4, 387-393.

Grubar, J. C., «Le sommeil paradoxal des débiles mentaux», comunicación presentada en el XXI Congreso Internacional de Psicología, París, 1976.

Grubar, J. C., «Le sommeil paradoxal des débiles mentaux», (en prensa).

Hartman, E., Les fonctions du sommeil, Dessart et Mardaga, 1975.

Hennevin, E. y Leconte, P., «La fonction du sommeil paradoxal: faits et hypothèses», Année Psychologique, 1971, 2, 491-519.

HeNRY, C. E., «Electroencephalograms of normal children», Monographs of Social Researcb on Children, Development National Research Council, Washington DC, 1944, II.

Hill, D., «The EEG in Psychiatry», en Electroencephalograpby. A synposium on its various aspects, D. Hill and Parr G. ed., Mc Millan, 1963, 368-428.

INHELDER, B., Le diagnostic du raisonnement cbez les débiles mentaux, Delachaux et Niestlé, 1959.

JACrson, J. H., Selected writings of John Hughlings Jackson (J. Taylor, ed.), Hodder and Stoughton, 1932.

JENKINS, J. y DaLLENBACH, K., «Obliviscence during sleep and waking», American Journal of Psychology, 1924, 35, 605-612.

JENSEN, A. R., "A theory of primary and secondary familial mental retardation», International Review of Research in Mental Retardation, 1970, 4, 33-105.

Jouvet, M., «Le discours biologique», Revue de médecine, 1972, 16, 1003-1063.

Jouvet, M., «Le rêve», La Recherche, 1974, 46, 515-517.

JouvET, M., «The role of monoaminergic neurors in the regulation and function of sleep», en Basic sleep mechanisms, Pètre-Quadens et Schlag ed., Academic Press, 1974, 207-236.

Jouvet, M. y Pujol, J. F., «Rôle des monoamines dans la régulation de la vigilance; étude neurophysiologique et biochimique», Revue de Neurologie, 1972, 127, 125-138.

Jouver, M., Miches, F. y CourJon, J., «Sur un stade d'activité électrique cérébrale rapide au cours du sommeil physiologique», Comptes Rendus de la Société de Biologie, París, 1959, $153,1024-1028$.

JouveT-Mounier, D., «Ontogénèse des états de vigilance chez quelques mammifères», tesis, Lyon, 1968.

LAJRY, G. C., «Données récentes sur la physiologie et la physiopathologie de l'activité onirique», Excerpta Medica International Congress Series, 1966, 117, 8-9.

LAIRY, G. C., «Données récentes sur la physiologie et la physiopathologie de l'activité onirique», en The abnormalities of sleep in man, Gastaut $\mathrm{H}$. $\mathrm{y}$ col., ed. Process of the xvth Europ meeting on Electroencephalography, Ardo Caggi, 1967, 275-283. 
Leconte, P., «Mise en évidence du rôle de la phase paradoxale du sommeil dans les processus de mémorisation», tesis, París, 1975.

LindSLEY, D. B., «Electrical potentials of the brain in children and adults», Jour of Genetic Psychology, 1938, 19, 285-306.

Loomis, A. L., Harvey, N. y Hobart, G. A., «Cerebral state during sleep, as studied by human brain potentiles», Journal of Experimental Psycbology, 1937, 21, 127-144.

MAIER, M. J. y MarTin, W. E., "Intellectual changes associated with Levo dopa therapy", Journal of the American Medical Association, 1970, 213, 465.

MiLner, B., «Psychological defects produced by temporal lobe excisions», en Readings in Pbysiological Psychology, Gross G. C. and Ziegler H. P. (eds.), Harper and Row, 1969, 156-169.

MoINET, C. y BenAGUE, $F$., «Les aptitudes rythmiques chez les débiles normaux et les débiles pathologiques», Mémoire pour le C.C.O., Lille, 1978.

Montpellier, de G., «Apprentissage et intelligence», Bulletin de Psychologie, 1976/77, 30 , $3 / 9,302-306$.

Mouret, J. R., Renaud, B., Quenin, P., Michel, D. y Schotr, B., «Monoamines et régulation de la vigilance. Apport et interprétation des données polygraphiques», Revue de Neurologie, 1972, 127, I, 138-155.

MoRUZzI, G., «General discussion», en Aspects anatomo fonctionnles de la physiologie du Sommeil (Jouvet M., ed.), CNRS, 1965, 638.

Mullers-KupPERs, M. y VoGel, F., «Uber die Personlichkeitsstruktur von Trägern einer seltener erblichen EEG variante», Psycbology, Psychotberapy, Medecin, Antbropology, 1965, $12,75-101$.

Netchine, S., «Eeg et intelligence Revue générale du problème», Année Psycbologique, 1959, 59 é, $427-438$.

NetChine, S., «Apports de la méthode longitudinale à l'étude de la maturation de l'électroencéphalogramme et de ses relations avec le développement psychologique de l'enfant normal», Enfance, 1967, 2, 175-178.

Netchine, S. y LatrX, G. C., «Ondes cérébrales et niveau mental. Quelques aspects de l'évolution génétique du tracé EEG suivant le niveau mental», Enfance, 1960, 4/5, 427-439.

Netchine, S., Talan, I., LajRY, G. C. y Zazzo, R., «Eeg et niveau mental: étude d'une population à niveau mental faible», Année Psycbologique, 1959, 59, 355-373.

Oswald, I., Sleep, Penguin Books, 1974.

Petre-Quadens, O., «On the different phases of the sleep of the new born with special reference to the activated phase or phased», Journal of Neurological Sciences, 1966, 3, 151-161.

Petre-Quadens, O., "Contribution à l'étude de la phase dite paradoxale du sommeil», Acta Neurologica Psychiatria Belgica, 1969, 769-898.

Petre-Quadens, O., «Sleep in mental retardation», en Sleep and the maturing nervous system, Academic Press, 1972, 383-417.

Petre-Quadens, O. y Jouvet, M., «Paradoxical sleep in mental retardation», Journal of Neurological Sciences, 1966, 3, 608-612.

Petre-Quadens, O. y DE Greef, A., «Effects of 5-HTP on sleep in mongol children», Journal of neurological sciences, 1971, 13, 115-119.

Piaget, J., La naissance de l'intelligence, Delachaux et Niestlé, 1936.

PIAGET, J., Biologie et connaissance, Gallimard, 1967 (trad. cast. Siglo XXI Ed. Madrid, 1970).

Piaget, J. e InHelder, B., Mémoire et intelligence, PUF, 1968.

Polikanina, R. I. y Sergeeva, L. N., «Development of extinctive inhibition in children in early ontogenesis», Zh. Vyssh. Deyat. Pavlova, 1967, 17, 228-229.

Rechtschaffen, A. y Kales, A., A manual of standardized terminology, tecbniques and scoring system for sleep stages of buman subjects, Public Health service US Government printing office, Washington, 1968.

Rhodes, L. E., Dustman, R. E. y Beck, E. C., «The visual evoked response: a comparison of bright and dull childrenm, Electroencephalography and clinical Neurophysiology, 1969, 27, $364-372$.

RoELANDT, J. L., «Corrélations entre tracés EEG de sommeil de nuit chez les enfants normaux et autistes», Mémoire de psycbiatrie, Lille, 1975.

Roffwarg, H., Muzio, J. y Dement, W., «Ontogenic development of human dream cycle», Science, 1966, 152, 604-619.

Roodin, P. A., Sullivan, L. y Rybash, J. M., «Effects of a memory aid on three types of conservation in institutionalized retarded children», Journal of Genetic Psychology, 1976, 129, 253-259.

Schmidt, H., Kaelbling, R. y Alexander, J., \&Sleep patterns in mental retardation: mongoloìds and monozygotic twinsm, Psychopbysiology, 1968, 5, 212. 
Sinson, J. C. y WETHERICE, N. E., \&Short term retention of colour and shape information in mongol and other severely subnormal children», Journal of Mental Deficiency Research, $1973,17,177$.

SMrrh, K. J., «Habituation of the orienting response to auditory stimulus sequences in the human new born», Condit. reflex., 1967, 2, 160-161.

SNYDER, F., "The new biology of dreaming», Archives of general psychiatry, 1963, 8 381-391.

Vanzulli, A., Cogacz, J., Handler, F. y García-Aust, T., «Evoked responses in man. I; Photic responses», Acta-neurologics Latina América 1960, 6, 303-309.

Voronin, L. G., Monovalov, V. F., Gromyko, M. M. y SERIKov, I. S., «Electrophysiological data on interaction of noticed and unnoticed trace processes in man in ontogenesisw, $Z h$. Vyssh. Nerv. Deyat. Pavlova, 1970, 20, 431-440.

Weiss, T. y Roldan, N. E., «Comparative study of sleep cycles in rodents», Experientia, 1964, $20,280-281$. 